\title{
Superconducting nanowire single-photon detector with ultralow dark count rate using cold optical filters
}

\author{
Hiroyuki Shibata $^{1,2}$, Kaoru Shimizu ${ }^{1}$, Hiroki Takesue ${ }^{1}$, and Yasuhiro Tokura ${ }^{1}$ \\ ${ }^{1}$ NTT Basic Research Laboratories, NTT Corporation, Atsugi-shi, Kanagawa 243-0198, Japan \\ ${ }^{2}$ Nanophotonics Center, NTT Corporation, Atsugi-shi, Kanagawa 243-0198, Japan
}

We report the fabrication of a superconducting nanowire single-photon detector (SSPD or SNSPD) with an ultralow dark count rate. By introducing optical band-pass filters at the input of the SSPD and cooling the filters at $3 \mathrm{~K}$, the dark count rate is reduced to less than 1/100 at low bias. An SSPD with 0.1 cps dark count rate and 5.6\% system detection efficiency at $1550 \mathrm{~nm}$ wavelength is obtained. We show that a quantum key distribution (QKD) over $300 \mathrm{~km}$ of fiber is possible based on a numerical calculation assuming a differential phase shift QKD protocol implemented with our SSPDs.

Superconducting single-photon detectors (SSPDs or SNSPDs) based on ultrathin superconducting nanowires are now widely used as high-performance single-photon detectors in many fields such as quantum information and

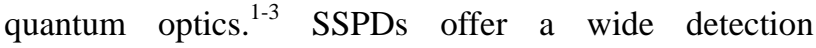
wavelength range from ultraviolet to infrared, a high detection efficiency $(\eta)$ of more than $10 \%$, a low dark count rate (DCR) of 10 to $1000 \mathrm{cps}$, and a small timing jitter $(\Delta \mathrm{t})$ of less than 100 ps. There have been many efforts to further improve their performance. In particular, many studies have been attempted to increase system $\eta$, and recently, a very high system $\eta$ of $93 \%$ has been reported using amorphous WSi. ${ }^{4-6}$ Besides $\eta$, the DCR is also an important factor determining the performance of single-photon detectors. The figure of merit of a single-photon detector is usually represented as $\eta /(\mathrm{DCR} \Delta \mathrm{t}) .^{7,8}$ In the figure of merit, decreasing DCR has the same weight as that of increasing $\eta$.

The importance of low DCR is also evident in long-distance quantum key distribution (QKD) experiments. ${ }^{9}$ In QKD, the quantum bit error rate (QBER) increases as the length of the fiber increases. A secure key cannot be obtained from the sifted key when QBER exceeds a certain level, which limits the QKD distance. Since the QBER is usually governed by the DCR of the detector, a single-photon detector with low DCR is essential for long-distance QKD. Actually, to achieve the QKD distance of $260 \mathrm{~km}$, the longest reported so far, SSPDs were operated in a very low-bias region to strongly reduce DCR $(\mathrm{DCR}=1 \mathrm{cps}, \eta=3 \%) .{ }^{10}$ To further increase the distance of QKD, an SSPD with lower DCR is necessary.

In this letter, we describe the fabrication of an SSPD with a dark count rate below 1 cps.

The fabrication process of the device is as follows. $^{11,12} \mathrm{NbN}$ thin films of $3.5 \mathrm{~nm}$ thickness were synthesized at $350^{\circ} \mathrm{C}$ on a $\mathrm{MgO}$ (100) substrate by reactive DC magnetron sputtering. The meander pattern of $\mathrm{NbN}$ was fabricated by the standard e-beam process using a negative resist and $\mathrm{C}_{2} \mathrm{~F}_{5}$ dry etching. The size of the meander is $10 \times 10 \mu \mathrm{m} 2$ with a line and space width of $80 \mathrm{~nm}$. Then, the cavity structure was formed on the meander using the standard photolithography process to enhance the detection efficiency. The cavity was 


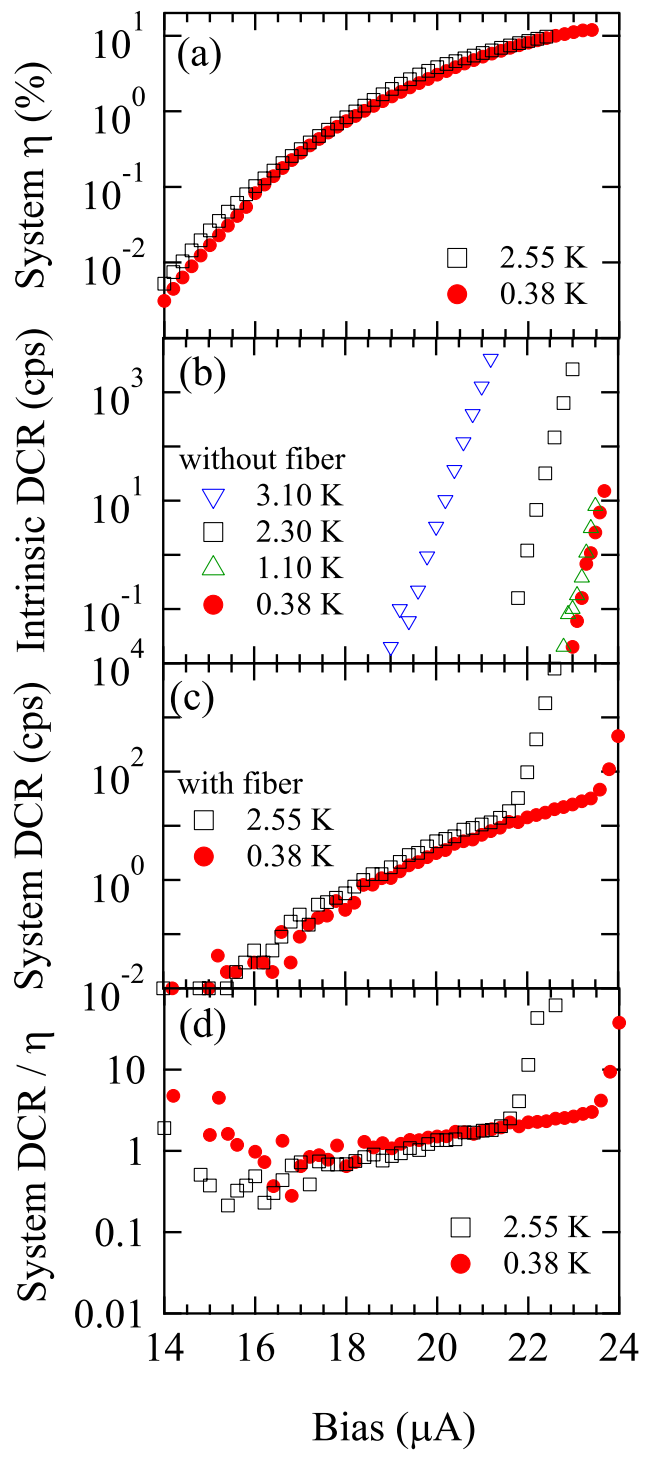

Fig. 1. Bias current dependences of (a) system detection efficiency $(\eta)$, (b) intrinsic dark count rate (DCR), (c) system DCR, and (d) system $D C R / \eta$ of the device at various temperatures.

composed of a 260-nm-thick $\mathrm{SiO}_{2}$ layer, 2-nm-thick Ti, and 100-nm-thick Au. A device with $\mathrm{T}_{\mathrm{c}}=10.5 \mathrm{~K}$ and $\mathrm{J}_{\mathrm{c}}=$ $5.3 \times 10^{6} \mathrm{~A} / \mathrm{cm}^{2}$ at $4.2 \mathrm{~K}$ was obtained by the above process.

For the optical characterization of the device, we used a ${ }^{3} \mathrm{He}$ cryocooler, which is able to cool the device down to $0.38 \mathrm{~K}$. The stability of the temperature is better than $5 \mathrm{mK}$ at the base temperature, while it increases to $50 \mathrm{mK}$ at high temperature $(0.5-3 \mathrm{~K})$. An external $50 \Omega$ shunt resistor was connected in parallel to the device on the cold stage and the signal was amplified using room-temperature $\mathrm{rf}$ amplifiers. A continuous-wave tunable laser with around $1550 \mathrm{~nm}$ wavelength was strongly attenuated and focused on the device to about 10 $\mu \mathrm{m}$ in diameter using a spherical lens at the end of the single-mode fiber. ${ }^{13,14}$ Transmission spectra of the band-pass filters were measured down to $\lambda=2.5 \mu \mathrm{m}$ by using a long-wavelength optical spectrum analyzer (YOKOGAWA AQ6375) and an incoherent white light source (Anritsu MG922A).

Figure 1(a) shows the bias current dependence of the system $\eta$ of the device. At $0.38 \mathrm{~K}$, the system $\eta$ increases as the bias current increases, and reaches $12 \%$ at the highest bias current of $23.4 \mu \mathrm{A}$. At $2.55 \mathrm{~K}$, almost the same line is followed, except that the highest efficiency is reduced to $9.4 \%$ at $22.4 \mu \mathrm{A}$. Figure 1(b) shows the bias current dependence of the DCR without connecting the optical fiber to the device, indicating the intrinsic DCR of the device. Although the intrinsic DCR is large in the high-bias-current region, it exponentially decreases as the bias current decreases and becomes below 0.1 cps. The curve shifts to the high-bias region as the temperature decreases and saturates below $1 \mathrm{~K}$. These features drastically change when an optical fiber is connected to the device without optical light input, as shown in Fig. 1(c). Although the exponential behavior is seen in the high-bias region, there appears a broad background in the low-bias region. As shown in Fig. 1(d), DCR/ $\eta$ is almost constant in the low-bias region, suggesting that the origin of the broad background is not the intrinsic property of the detector. This broad background comes from blackbody radiation at room temperature, which propagates through the optical fiber. This indicates that the system DCR is governed by the blackbody DCR in the low-bias region. These features have also been reported in ref. 15.

The DCR due to a single-mode blackbody cavity at 

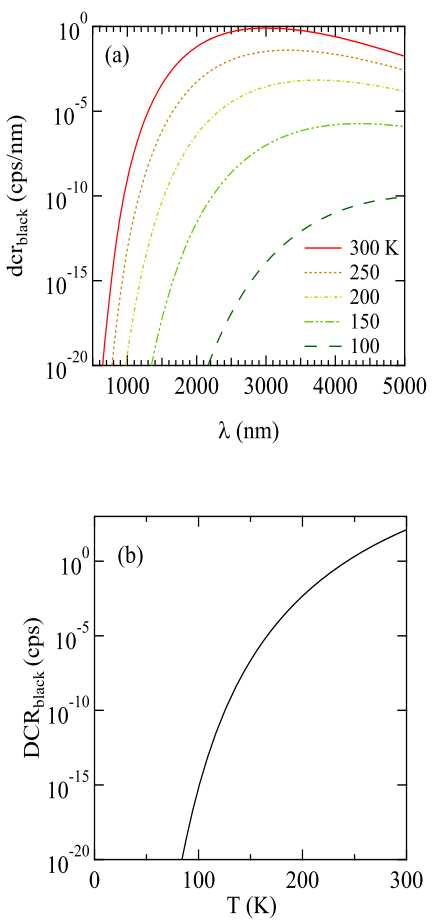

Fig. 2. (a) Calculated dark count rate $\left(\mathrm{dcr}_{\text {black }}\right)$ per nanometer wavelength due to blackbody radiation using eq. (1), and (b) $\operatorname{DCR}_{\text {black }}(T)$ calculated by integrating dcr $_{\text {black }}$ between 200 to $2500 \mathrm{~nm}$.

temperature $\mathrm{T}$ through single-mode optical fiber is approximately expressed as

$$
\begin{aligned}
\operatorname{DCR}_{\text {black }}\left(\lambda_{0}, \lambda_{1}, T\right) & =\int_{\lambda_{1}}^{\lambda_{0}} d c r_{\text {black }}(\lambda, T) d \lambda \\
& =\int_{\lambda_{1}}^{\lambda_{0}} \frac{2 c \varepsilon \eta}{\lambda^{2}} \frac{1}{e^{h c / \lambda k_{B} T}-1} d \lambda
\end{aligned}
$$

where $\mathrm{dcr}_{\text {black }}(\lambda, T)$ is the dark count rate per nanometer wavelength, $\varepsilon$ is emissivity of the cavity, and $\lambda_{0}$ and $\lambda_{1}$ are the long- and short-wavelength absorption edges of the fiber. ${ }^{16,17}$ Figure 2 shows wavelength dependence of $\operatorname{dcr}_{\text {black }}(\lambda, T)$ at various temperatures, calculated using eq. (1). In the calculation, we assume that $\varepsilon=1$ for all wavelengths, $\eta=10^{2} \exp \left(-2 \times \ln 10 \times 10^{6} \times \lambda\right)$ below $\lambda=$ $1000 \mathrm{~nm}$, and $\eta=1$ above $\lambda=1000 \mathrm{~nm}^{2}$ At $300 \mathrm{~K}$, $\mathrm{dcr}_{\text {black }}$ takes the maximum at around $\lambda=3000 \mathrm{~nm}$ and exponentially decreases at short wavelength. As the
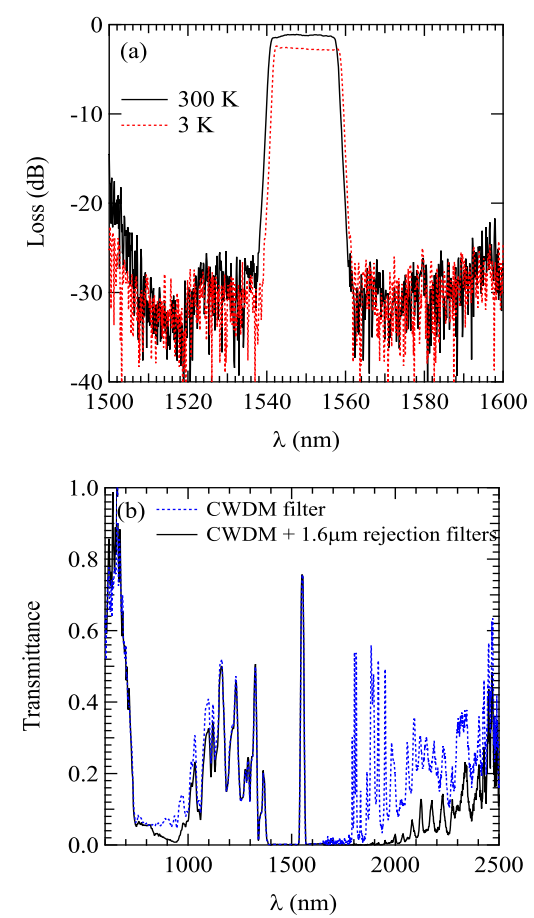

Fig. 3. (a) Transmission characteristics of the band-pass filters introduced at the input of the device at room temperature and at 3 K. (b) Transmission characteristics of band-pass filters (solid line) and only CWDM filter (dashed line) at room temperature in a wide wavelength region.

temperature decreases, dcr $r_{\text {black }}$ strongly decreases and becomes negligible below $\mathrm{T}<100 \mathrm{~K}$. The inset of Fig. 2 shows the calculated temperature dependences of

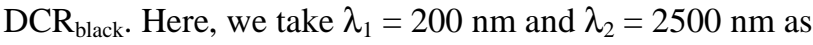
the transmission range of the $1.5 \mu \mathrm{m}$ single-mode fiber. At $300 \mathrm{~K}, \mathrm{DCR}_{\text {black }}$ becomes $1.28 \times 10^{2} \mathrm{cps}$, which is comparable to the observed DCR in Fig. 1(c), but it exponentially decreases as temperature decreases. This indicates that DCR from the blackbody can be removed by introducing an optical band-pass filter and cooling it below $100 \mathrm{~K}$.

For the cold band-pass filters, a coarse wavelength division multiplexer (CWDM) filter and $1.6 \mu \mathrm{m}$ rejection short-wavelength pass filter, which are commercially available, are used. ${ }^{18}$ They are connected in series and are cooled at the second stage of the cryocooler cooled to $3 \mathrm{~K}$. 


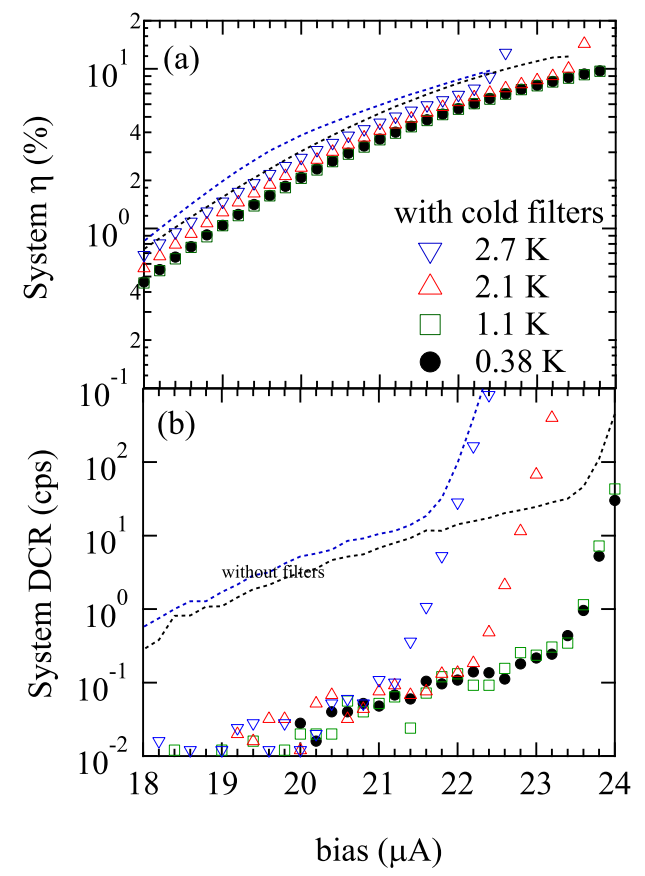

Fig. 4. Bias current dependence of (a) system detection efficiency $(\eta)$ and (b) system dark count rate (DCR) with filter that was cooled to $3 \mathrm{~K}$ at various device temperatures. The thin dashed lines are without cold filters at device temperatures of 0.38 (black) and $2.55 \mathrm{~K}$ (blue).

Figure 3(a) shows the transmission properties of the filters. They have a bandwidth of $20 \mathrm{~nm}$ at $1550 \mathrm{~nm}$ wavelength and insertion loss of $1.2 \mathrm{dBm}$ at room temperature, including the coupling loss at the cryocooler. As the temperature decreases to $3 \mathrm{~K}$, the insertion loss increases to $2.7 \mathrm{dBm}$ and the pass-band shifts about $1 \mathrm{~nm}$. Broadband transmission properties of the filters are shown in Fig. 3(b). The long-wave blocking limit is about $1800 \mathrm{~nm}$ for a single CWDM filter, and it is extended to $2000 \mathrm{~nm}$ by adding a $1.6 \mu \mathrm{m}$ rejection filter.

Figure 4 shows (a) system $\eta$ and (b) system DCR of the device with cold filters at $3 \mathrm{~K}$. The system $\eta$ decreases to $60 \%$ due to the loss of the filters. Although the system DCR strongly increases in the high-bias region, it strongly decreases in the low-bias region due to the protection of blackbody radiation. At a bias current of $22.0 \mu \mathrm{A}$ and at $0.38 \mathrm{~K}$, system $\eta$ decreases from 8.1 to

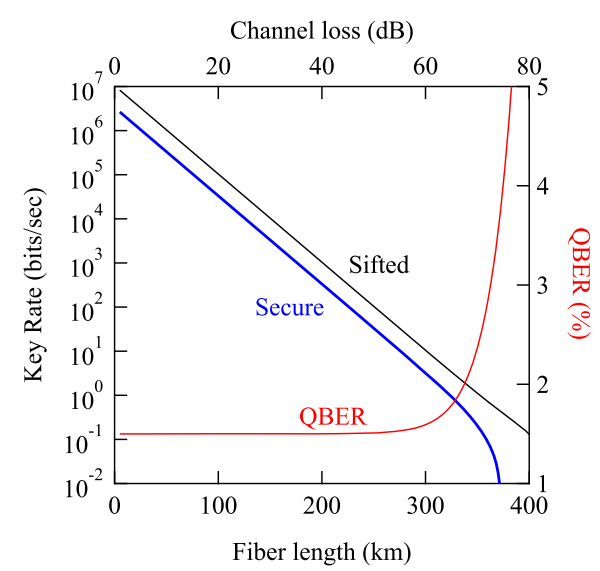

Fig. 5. Calculated fiber length dependences of key rate and QBER using the ultralow dark count SSPD $(\eta=5 \%$, DCR $=0.1$ cps, $1 \mathrm{GHz}$ clock speed, 100 ps time windows, DPS-QKD protocol, $0.2 \mathrm{~dB} / \mathrm{km}$ fiber loss).

5.6\%, while system DCR decreases from 14 to 0.1 cps by the insertion of cold filters, and the figure of merit increases 97 -fold. This clearly shows the importance of cold filters to the figure of merit. It is noted that such a high increase of the figure of merit cannot be expected by increasing $\eta$ of the device without insertion of cold filters, since $D R_{\text {black }}$ also increases with increasing $\eta$. The strong decrease of system DCR is also observed at $2.7 \mathrm{~K}$, which can be reached using the standard Gifford-McMahon cryocooler. At a bias current of 21.0 $\mu \mathrm{A}$ and at $2.7 \mathrm{~K}$, system $\eta$ of $4.6 \%$ and system DCR of 0.1 cps are obtained by the insertion of cold filters.

Although the DCR is strongly reduced by the cold filters, there remains a broad background on the order of $0.1 \mathrm{cps}$ in the low-bias region in Fig. 4(b). This is due to the remaining blackbody radiation that passes through the cold filters. As shown in Fig. 3(b), there are three main pass-bands: shorter than $1400 \mathrm{~nm}, 1540$ to $1560 \mathrm{~nm}$, and longer than $2000 \mathrm{~nm}$. Since dcr black increases at the longer wavelength, the main source of residual DCR comes from the pass-band longer than $2000 \mathrm{~nm}$. Actually, we confirmed that system DCR decreases to only $1 / 5$ with the CWDM filter alone. It seems possible to further 
reduce the $\mathrm{DCR}_{\text {black }}$ by using a cold filter with a wider rejection-wavelength region.

Figure 5 shows the calculation of the fiber length dependence of key rate and QBER using the above SSPD. In the calculation, we assume a standard telecom fiber with the loss coefficient of $0.2 \mathrm{~dB} / \mathrm{km}$, the differential phase shift QKD (DPS-QKD) protocol under general individual attacks, a $1 \mathrm{GHz}$ clock rate, 100 ps time windows, $\eta=5 \%$, and DCR $=0.1 \mathrm{cps} .{ }^{19,20}$ In the figure, the sifted key rate exponentially decreases as the fiber length increases. The secure key rate also exponentially decreases but it drops at about $350 \mathrm{~km}$ due to the strong increase of QBER. The secure key rate is $2.5 \mathrm{bit} / \mathrm{s}$ at 300 $\mathrm{km}$, which is enough for secure communications.

In conclusion, we report the fabrication of SSPD with an ultralow dark count rate. The main origin of the dark count rate in the low-bias region is blackbody radiation at room temperature, which propagates through optical fiber. By introducing cold band-pass filters at the input of the SSPD, the dark count rate can be strongly reduced. An SSPD with a 0.1 cps dark count rate and $5.6 \%$ system detection efficiency is obtained, which can be used in QKD over a distance of more than $300 \mathrm{~km}$.

1) G. N. Gol’tsman, O. Okunev, G. Chulkova, A. Lipatov, A. Semenov, K. Smirnov, B. Voronov, A. Dzardanov, C. Williams, and R. Sobolewski: Appl. Phys. Lett. 79 (2001) 705.

2) A. Korneev, A. Divochiy, Y. Vachtomin, Y. Korneeva, I. Florya, M. Elezov, N. Manova, M. Tarkhov, P. An, A. Kardakova, A. Isupova, G. Chulkova, K. Smirnov, N. Kaurova, V. Seleznev, B. Voronov, and G. Goltsman: Proc. SPIE 8072 (2011) 807202.

3) C. M. Natarajan, M. G. Tanner, and R. H. Hadfield: Supercond. Sci. Technol. 25 (2012) 063001.

4) D. Rosenberg, A. J. Kerman, R. J. Molnar, and E. A. Dauler:
Opt. Express 21 (2013) 1440.

5) F. Marsili, V. B. Verma, J. A. Stern, S. Harrington, A. E. Lita, T. Gerrits, I. Vayshenker, B. Baek, M. D. Shaw, R. P. Mirin, and S. W. Nam: Nat. Photonics 7 (2013) 210.

6) S. Miki, T. Yamashita, H. Terai, and Z. Wang: Opt. Exp. 21 (2013) 10208.

7) R. H. Hadfield: Nat. Photonics 3 (2009) 696.

8) M. D. Eisaman, J. Fan, A. Migdall, and S. V. Polyakov: Rev. Sci. Instrum. 82 (2011) 071101.

9) H. Takesue, S. W. Nam, Q. Zhang, R. H. Hadfield, T. Honjo, K. Tamaki, and Y. Yamamoto: Nat. Photonics 1 (2007) 343.

10) S. Wang, W. Chen, J. F. Guo, Z. Q. Yin, H. W. Li, Z. Zhou, G. C. Guo, and Z. F. Han: Opt. Lett. 37 (2012) 1008.

11) T. Seki, H. Shibata, H. Takesue, Y. Tokura, and N. Imoto: Physica C 470 (2010) 1534.

12) H. Shibata, N. Kaina, T. Seki, Y. Tokura, and N. Imoto: Physics Procedia 36 (2012) 324.

13) H. Shibata, H. Takesue, T. Honjo, T. Akazaki, and Y. Tokura: Appl. Phys. Lett. 97 (2010) 212504.

14) H. Shibata, T. Akazaki, and Y. Tokura: Appl. Phys. Express 6 (2013) 023101.

15) T. Yamashita, S. Miki, W. Qui, M. Fujiwara, M. Sasaki, and Z. Wang: Appl. Phys. Express 3 (2010) 102502.

16) A. J. Miller, A. Lita, D. Rosenberg, S. Gruber, and S. W. Nam: Proc. 8th Int. Conf. on Quantum Communication, Measurement and Computing, 2007, p. 445.

17) D. Fukuda, G. Fujii, T. Numata, K. Amemiya, A. Yoshizawa, H. Tsuchida, H. Fujino, H. Ishii, T. Itatani, S. Inoue, and T. Zama: IEEE Trans. Appl. Supercond. 21 (2011) 241.

18) CWDM-55 (Advanced Fiber Resources Ltd.) and 1550/1625 SWPF (Sumitomo Electric Ltd.).

19) K. Inoue, E. Waks, and Y. Yamamoto: Phys. Rev. Lett. 89 (2002) 037902.

20) E. Waks, H. Takesue, and Y. Yamamoto: Phys. Rev. A 73

(2006) 012344. 